\title{
$K$-UNIFORM ROTUNDITY OF SEQUENCE ORLICZ SPACES
}

\author{
WANG TINGFU AND CHEN SHUTAO
}

\begin{abstract}
This paper presents a criterion of KUR for sequence Orlicź spaces with Luxemburg's norm. The result also indicates that for any integer $k \geq 1$, there exists a $k+1$-uniformly rotund Banach space not being $k$-uniformly rotund.
\end{abstract}

Let $M:(-\infty,+\infty) \rightarrow[0,+\infty)$ be convex, even, continuous and $M(u)=0 \Leftrightarrow u=0$. For a given sequence $x=\left(x_{n}\right)$, denote $\rho(x)=\sum_{n} M\left(x_{n}\right)$ and $\ell_{M}=\left\{x=\left(x_{n}\right): \exists \lambda>0\right.$, $\rho(\lambda x)<\infty\},\|x\|=\inf \{\lambda>0: \rho(x / \lambda) \leq 1\}$ for $x \in \ell_{M}$, then $\left(\ell_{M},\|\|\right)$ is a Banach space.

We say $M \in \delta_{2}$, if $\varlimsup_{u \rightarrow 0} M(2 u) / M(u)<\infty$,

LEMMA 1 [2,3]. If $M \in \delta_{2}$, then

(a) $\left\|x^{n}\right\| \rightarrow 0 \Leftrightarrow \rho\left(x^{n}\right) \rightarrow 0$,

(b) $\left\|x^{n}\right\| \rightarrow 1 \Leftrightarrow \rho\left(x^{n}\right) \rightarrow 1$ and

(c) for any $1>0, \epsilon>0$, there exists $\delta>0$ such that $\rho(x) \leq 1, \rho(y) \leq \delta$ imply

$$
|\rho(x+y)-\rho(x)|<\epsilon
$$

$M(u)$ is said to be uniformly convex on some interval $[a, b]$, if for any $\epsilon>0$, there exists $\delta>0$ such that $u, v$ in $[a, b],|u-v| \geq \epsilon \max \{u, v\}$ imply

$$
M\left[\frac{1}{2}(u+v)\right] \leq \frac{1}{2}(1-\delta)[M(u)+M(v)]
$$

LEMMA 2. The following are equivalent

(I) $M(u)$ is uniformly convex on $[0, a]$,

(II) for any $\beta \in[0, a), b \geq a$ and $\epsilon>0$, there exists $\delta>0$ such that $\max \{u, v\} \leq$ $b, 0 \leq \min \{u, v\} \leq \beta$ and $|u-v| \geq \epsilon \max \{u, v\}$ imply

$$
M\left[\frac{1}{2}(u+v)\right] \leq \frac{1}{2}(1-\delta)[M(u)+M(v)]
$$

(III) for any integer $m \geq 2, \beta \in[0, a), b \geq a$ and $\epsilon>0$, there exists $\delta>0$ such that $\max _{1 \leq j \leq m} u_{j} \leq b, 0 \leq \min _{1 \leq j \leq m} u_{j} \leq \beta$ and $\max _{1 \leq i, j \leq m}\left|u_{i}-u_{j}\right| \geq \epsilon \max _{1 \leq j \leq m} u_{j}$ imply

$$
M\left(\sum_{j} u_{j} / m\right) \leq(1-\delta) \sum_{j} M\left(u_{j}\right) / m
$$

Received by the editors March 2, 1988, revised December 20, 1988.

AMS subject classification: Primary: 46B30, Secondary: 46B20.

(C) Canadian Mathematical Society 1991. 
Proof. (I) $\Rightarrow$ (II). If (2) is not true, then there exist $\beta<a, b \geq a, \epsilon>0$ and $u_{n}$, $v_{n} \leq b, u_{n}-v_{n} \geq \epsilon u_{n}>0$ such that

$$
M\left[\frac{1}{2}\left(u_{n}+v_{n}\right)\right]>\frac{1}{2}\left(1-\frac{1}{n}\right)\left[M\left(u_{n}\right)+M\left(v_{n}\right)\right] \quad(n=1,2, \ldots) .
$$

Without loss of generality, we may assume $u_{n} \rightarrow u^{\prime}$ and $v_{n} \rightarrow v^{\prime}$; then by (I), $u^{\prime} \geq a>$ $\beta \geq v^{\prime}$ and from (1) it is easy to see $M\left[\frac{1}{2}\left(u^{\prime}+v^{\prime}\right)\right]=\frac{1}{2}\left[M\left(u^{\prime}\right)+M\left(v^{\prime}\right)\right]$ which shows that $M(u)$ is linear on $\left[v^{\prime}, u^{\prime}\right]$ contradicting (I).

(II) $\Rightarrow$ (III). For given $\beta\langle a, b \geq a$ and $\epsilon>0$, select $\delta>0$ satisfying (II). For any integer $m \geq 2$ and real numbers $\left\{u_{j}\right\}$ satisfying the conditions in (III), we may assume $u_{1}=\max _{1 \leq j \leq m} u_{j}, u_{2}=\min _{1 \leq j \leq m} u_{j}$.

If $m$ is even, then

$$
\begin{aligned}
M\left[\left(u_{1}+\cdots+u_{m}\right) / m\right] & =M\left\{\left[\frac{1}{2}\left(u_{1}+u_{2}\right)+\cdots+\frac{1}{2}\left(u_{m-1}+u_{m}\right)\right] /(m / 2)\right\} \\
& \leq(2 / m)\left\{M\left[\frac{1}{2}\left(u_{1}+u_{2}\right)\right]+\cdots+M\left[\frac{1}{2}\left(u_{m-1}+u_{m}\right)\right]\right\} \\
& \leq(2 / m)\left\{\frac{1}{2}(1-\delta)\left[M\left(u_{1}\right)+M\left(u_{2}\right)\right]+\frac{1}{2}\left[M\left(u_{3}\right)+M\left(u_{4}\right)\right]\right. \\
& \left.+\cdots+\frac{1}{2}\left[M\left(u_{m-1}\right)+M\left(u_{m}\right)\right]\right\} \\
& =\sum_{j} M\left(u_{j}\right) / m-\delta\left[M\left(u_{1}\right)+M\left(u_{2}\right)\right] / m \\
& \leq \sum_{j} M\left(u_{j}\right) / m-\delta \sum_{j} M\left(u_{j}\right) / m^{2} \\
& =(1-\delta / m) \sum_{j} M\left(u_{j}\right) / m
\end{aligned}
$$

If $m$ is odd, observe $u_{1}>\sum_{j} u_{j} / m>u_{2}$, by the result we just obtained, we have

$$
\begin{aligned}
M\left[\left(u_{1}\right.\right. & \left.\left.+\cdots+u_{m}\right) / m\right] \\
& =M\left\{\left[\left(u_{1}+\cdots+u_{m}+\left(u_{1}+\cdots+u_{m}\right) / m\right] /(m+1)\right\}\right. \\
& \leq[1-\delta /(m+1)]\left\{\sum_{j} M\left(u_{j}\right)+M\left[\left(u_{1}+\cdots+u_{m}\right) / m\right]\right\} /(m+1) \\
& \leq[1-\delta /(m+1)] \sum_{j} M\left(u_{j}\right) / m
\end{aligned}
$$

(III) $\Rightarrow(\mathrm{I})$. In fact, (I) is the special case of (III) when $m=2, \beta=(1-\epsilon) a$ and $b=a$.

Now, we turn to the $k$-uniform rotundity. A Banach space $X$ is $k$-uniformly rotund, provided that for any $\epsilon>0$, there exists $\delta>0$ such that for any $x^{1}, \ldots, x^{k+1} \in S(X)$, the sphere of $X, \Delta\left(x^{1}, \ldots, x^{k+1}\right) \geq \epsilon$ implies $\left\|x^{1}+\cdots+x^{k+1}\right\| \leq(1-\delta)(k+1)$, where

(see [1]).

$$
\Delta\left(x^{1}, \ldots, x^{k+1}\right)=\sup _{f_{j} \in S\left(X^{*}\right)}\left|\begin{array}{cccc}
1 & 1 & \cdots & 1 \\
f_{1}\left(x^{1}\right) & f_{1}\left(x^{2}\right) & \cdots & f_{1}\left(x^{k+1}\right) \\
\vdots & \vdots & \vdots & \vdots \\
f_{k}\left(x^{1}\right) & f_{k}\left(x^{2}\right) & \cdots & f_{k}\left(x^{k+1}\right)
\end{array}\right|
$$


THEOREM 1. $M$ is $k$-uniformly rotund $(k \geq 1)$ if and only if $(a) M \in \delta_{2}$ and $(b) M(u)$ is uniformly convex on $\left[0, M^{-1}(1 /(k+1))\right]$.

PROOF. Necessity: $(a)$ is trivial since $k$-uniform rotundity implies reflexivity. If $(b)$ does not hold, then there exists $\epsilon>0, u_{n}, v_{n} \in\left[0, M^{-1}(1 /(k+1))\right], u_{n}-v_{n} \geq \epsilon u_{n}$ satisfying

$$
M\left[\frac{1}{2}\left(u_{n}+v_{n}\right)\right]>\frac{1}{2}\left(1-\frac{1}{n}\right)\left[M\left(u_{n}\right)+M\left(v_{n}\right)\right] \quad(n=1,2, \ldots) .
$$

Denote $w_{n}=\left[(k-1) u_{n}+(k+1) v_{n}\right] / 2 k$, then $M\left(v_{n}\right)<M\left(w_{n}\right)<M\left(u_{n}\right) \leq 1 /(k+$ $1)$, therefore, $M\left(u_{n}\right)+k M\left(w_{n}\right)<1$. Choose an integer $m_{n}$ such that $\frac{1}{2}<m_{n}\left[M\left(u_{n}\right)+\right.$ $\left.k M\left(w_{n}\right)\right] \leq 1$ and $t_{n} \geq 0$ such that $m_{n}\left[M\left(u_{n}\right)+k M\left(w_{n}\right)\right]+M\left(t_{n}\right)=1$, define

$$
\begin{aligned}
x^{(n, 1)} & =(\overbrace{w_{n}, \ldots, w_{n}}^{k m_{n}}, \overbrace{u_{n}, \ldots, u_{n}}^{m_{n}}, t_{n}, 0,0, \ldots) \\
x^{(n, 2)} & =\overbrace{\left(u_{n}, \ldots, u_{n}\right.}^{m_{n}}, \overbrace{w_{n}, \ldots, w_{n}}^{k m_{n}}, t_{n}, 0,0, \ldots) \\
x^{(n, 3)} & =(\overbrace{w_{n}, \ldots, w_{n}}^{m_{n}}, \overbrace{u_{n}, \ldots, u_{n}}^{m_{n}}, \overbrace{w_{n}, \ldots, w_{n}}^{(k-1) m_{n}}, t_{n}, 0,0, \ldots) \\
& \vdots \\
x^{(n, k)} & =\overbrace{\left(w_{n}, \ldots, w_{n}\right.}^{(k-2) m_{n}}, \overbrace{u_{n}, \ldots, u_{n}}^{m_{n}}, \overbrace{w_{n}, \ldots, w_{n}}^{2 m_{n}}, t_{n}, 0,0, \ldots) \\
x^{(n, k+1)} & =(\overbrace{w_{n}, \ldots, w_{n}}^{m_{n}}, \overbrace{u_{n}, \ldots, u_{n}}^{m_{n}}, \overbrace{w_{n}, \ldots, w_{n}}^{2 m_{n}}, t_{n}, 0,0, \ldots) \quad(n=1,2, \ldots),
\end{aligned}
$$

then for each $n \geq 1$ and $j \leq k+1, \rho\left(x^{(n, j)}\right)=m_{n} M\left(u_{n}\right)+k m_{n} M\left(w_{n}\right)+M\left(t_{n}\right)=1$; therefore, $\left\|x^{(n, j)}\right\|=1$. Furthermore

$$
\begin{aligned}
& \rho\left(\sum_{j} x^{n, j} /(k+1)\right)=(k+1) m_{n} M\left[\left(u_{n}+k w_{n}\right) /(k+1)\right]+M\left(t_{n}\right) \\
& \quad=(k+1) m_{n} M\left[\frac{1}{2}\left(u_{n}+v_{n}\right)\right]+M\left(t_{n}\right) \\
& \quad>(k+1) m_{n}\left(1-\frac{1}{n}\right) \frac{\left[M\left(u_{n}\right)+M\left(v_{n}\right)\right]}{2}+M\left(t_{n}\right) \\
& \quad=\left(1-\frac{1}{n}\right)\left\{m_{n} M\left(u_{n}\right)+k m_{n} \frac{\left[(k-1) M\left(u_{n}\right)+(k+1) M\left(v_{n}\right)\right]}{2 k}+M\left(t_{n}\right)\right\} \\
& \geq\left(1-\frac{1}{n}\right)\left\{m_{n} M\left(u_{n}\right)+k m_{n} M\left(w_{n}\right)+M\left(t_{n}\right)\right\} \\
& \quad=\left(1-\frac{1}{n}\right) \rightarrow 1
\end{aligned}
$$

it follows $\left\|\sum_{j} x^{(n, j)}\right\| \rightarrow k+1,(n \rightarrow \infty)$. 
Set $c_{n}=\left[m_{n} M^{-1}\left(\frac{1}{m} n\right)\right]^{-1}$ and

$$
f^{(n, j)}=(\overbrace{0, \ldots, 0}^{(j-1) m_{n}}, \overbrace{c_{n}, \ldots, c_{n}}^{m_{n}} 0,0, \ldots) \quad(n=1,2, \ldots ; j=1,2, \ldots, k)
$$

then $f^{(n, j)} \in \ell_{M}^{*}$ and $\left\|f^{(n, j)}\right\|=1 \quad(n=1,2, \ldots ; j=1,2, \ldots, k),($ see $[4,5])$ thus

$$
\begin{aligned}
& \Delta\left(x^{(n, 1)}, \ldots, x^{(n, k+1)}\right) \geq\left|\begin{array}{cccc}
1 & 1 & \cdots & 1 \\
f^{(n, 1)}\left(x^{(n, 1)}\right) & f^{(n, 1)}\left(x^{(n, 2)}\right) & \cdots & f^{(n, 1)}\left(x^{(n, k+1)}\right) \\
\vdots & \vdots & \vdots & \vdots \\
f^{(n, k)}\left(x^{(n, 1)}\right) & f^{(n, k)}\left(x^{(n, 2)}\right) & \cdots & f^{(n, k)}\left(x^{(n, k+1)}\right)
\end{array}\right| \\
& =\left|\begin{array}{ccccc}
c_{n} m_{n}\left(u_{n}-w_{n}\right) & 0 & 0 & \cdots & 0 \\
0 & c_{n} m_{n}\left(u_{n}-w_{n}\right) & 0 & \cdots & 0 \\
\vdots & \vdots & \vdots & \vdots & \vdots \\
0 & 0 & 0 & \cdots & c_{n} m_{n}\left(u_{n}-w_{n}\right)
\end{array}\right| \\
& =\left[c_{n} m_{n}\left(u_{n}-w_{n}\right)\right]^{k} \\
& =\left[c_{n} m_{n}(k+1)\left(u_{n}-v_{n}\right) / 2 k\right]^{k} \\
& \geq\left[(k+1) \epsilon c_{n} m_{n} u_{n} / 2 k\right]^{k}
\end{aligned}
$$

By $(1), m_{n}(k+1) M\left(u_{n}\right)>\frac{1}{2}$, therefore

$$
\begin{aligned}
c_{n} m_{n} u_{n} & =u_{n} / M^{-1}\left(1 / m_{n}\right) \\
& >u_{n} / M^{-1}\left[2(k+1) M\left(u_{n}\right)\right] \\
& >\frac{1}{2(k+1)}
\end{aligned}
$$

Thus,

$$
\begin{gathered}
\Delta\left(x^{(n, 1)}, \ldots, x^{(n, k+1)}\right) \geq[(k+1) \epsilon / 4 k(k+1)]^{k} \\
=(\epsilon / 4 k)^{k}
\end{gathered}
$$

a contradiction.

Sufficiency: let $x^{(n, 1)}, \ldots, x^{(n, k+1)} \epsilon S\left(\ell_{M}\right)$ and $\rho\left[\frac{\sum_{j} x^{(n, j)}}{(k+1)}\right] \rightarrow 1$ as $n \rightarrow \infty$. We need to prove that for any $\tau>0$, we have $\Delta_{n} \equiv \Delta\left(x^{(n, 1)}, \ldots, x^{(n, k+1)}\right) \leq O(\tau)$ for all $n$ large enough.

By Lemma 1, we can select $\epsilon>0$ satisfying $(k+1) \epsilon<1$ and

$$
\begin{aligned}
& \rho(x) \leq 1, \rho(y) \leq(k+2) \epsilon \Rightarrow|\rho(x+y)-\rho(x)|<\tau, \\
& \rho(z) \leq(k+1) \epsilon \Rightarrow|| z \|<\tau .
\end{aligned}
$$

Choose $\beta \in\left(0, M^{-1}\left[\frac{1}{(k+1)}\right]\right)$ such that

$$
k M(\beta)+M[(1+\epsilon) \beta]>1
$$


and denote

$$
\begin{aligned}
& I_{n}=\left\{i: \max _{j, 1}\left\{\left|x_{i}^{(n, j)}-x_{i}^{(n, 1)}\right|\right\}<\epsilon \max _{j}\left|x_{i}^{(n, j)}\right|\right\} \\
& J_{n}=\left\{i: \max _{j, 1}\left\{\left|x_{i}^{(n, j)}-x_{i}^{(n, 1)}\right|\right\} \geq \epsilon \max _{j}\left|x_{i}^{(n, j)}\right|\right\} ; \quad \text { or }\left(x_{i}^{(n, j)}\right)_{j}
\end{aligned}
$$

have different signs or they have same sign but $\left.\min _{j}\left|x_{i}^{(n, j)}\right| \leq \beta\right\}$

$$
K_{n}=\left\{i: \max _{j, 1}\left\{\left|x_{i}^{(n, j)}-x_{i}^{(n, 1)}\right|\right\} \geq \epsilon \max _{j}\left|x_{i}^{(n, j)}\right|\right\} ;\left(x_{i}^{(n, j)}\right)_{j}
$$

$$
\text { have same sign and } \left.\max _{j}\left|x_{i}^{(n, j)}\right|>\beta\right\} \quad(n=1,2, \ldots) .
$$

Since $M \in \delta_{2}$, for $f_{n} \in S\left(\ell_{M}^{*}\right)$, there exists $\left(y_{i}^{(n)}\right)_{i} \in \ell_{N}$ such that $f_{n}(x)=\sum_{i} x_{i} y_{i}^{(n)}$, where $N(v)=\sup _{u}\{u v-M(u)\}($ see[4,5]). Therefore

$$
\begin{aligned}
& \Delta_{n}=\sup _{\substack{\left\|y^{j}\right\|_{N} \leq 1 ; \\
(j=1, \ldots, k)}}\left|\begin{array}{ccc}
\sum_{i} y_{i}^{(1)}\left(x_{i}^{(n, 2)}-x_{i}^{(n, 1)}\right) & \cdots & \sum_{i} y_{i}^{(1)}\left(x_{i}^{(n, k+1)}-x_{i}^{(n, 1)}\right) \\
\vdots & \vdots & \vdots \\
\sum_{i} y_{i}^{(k)}\left(x_{i}^{(n, 2)}-x_{i}^{(n, 1)}\right) & \cdots & \sum_{i} y_{i}^{(k)}\left(x_{i}^{(n, k+1)}-x_{i}^{(n, 1)}\right)
\end{array}\right| \\
&=\sup \left|\begin{array}{ccc}
\sum_{I_{n}+J_{n}+H_{n}} y_{i}^{(1)}\left(x_{i}^{(n, 2)}-x_{i}^{(n, 1)}\right) & \cdots & \sum_{I_{n}+J_{n}+H_{n}} y_{i}^{(1)}\left(x_{i}^{(n, k+1)}-x_{i}^{(n, 1)}\right) \\
\vdots & \vdots & \vdots \\
\sum_{I_{n}+J_{n}+H_{n}} y_{i}^{(k)}\left(x_{i}^{(n, 2)}-x_{i}^{(n, 1)}\right) & \cdots & \sum_{I_{n}+J_{n}+H_{n}} y_{i}^{(k)}\left(x_{i}^{(n, k+1)}-x_{i}^{(n, 1)}\right)
\end{array}\right|
\end{aligned}
$$

Denote $e^{i}=(0, \ldots, 0, \overbrace{1}^{i \text { th }}, 0, \ldots)$. By the choice of $I_{n}$ and $(K+1) \epsilon<1$, we have

(6)

$$
\begin{aligned}
\left.\rho\left(\sum_{I_{n}}\left(x_{i}^{(n, j)}-x_{i}^{(n, 1)}\right) e^{i}\right)\right) & =\sum_{I_{n}} M\left(x_{i}^{(n, j)}-x_{i}^{(n, 1)}\right) \\
& \leq \sum_{I_{n}} M\left(\epsilon\left(\left|x_{i}^{(n, 1)}\right|+\cdots+\left|x_{i}^{(n, k+1)}\right|\right)\right) \\
& <(k+1) \epsilon \sum_{I_{n}} M\left(\frac{\sum_{j}\left|x_{i}^{(n, j)}\right|}{(k+1)}\right) \\
& \leq \epsilon \sum_{j} \sum_{i} M\left(x_{i}^{(n, j)}\right) \\
& =(k+1) \epsilon \quad(j=1, \ldots, k+1)
\end{aligned}
$$

It follows from (3) that $\left\|\left(\sum_{I_{n}}\left(x_{i}^{(n, j)}-x_{i}^{(n, 1)}\right) e^{i}\right)\right\|<\tau$, therefore

$$
\begin{aligned}
\left|\sum_{I_{n}} y_{i}^{(h)}\left(x_{i}^{(n, j)}-x_{i}^{(n, 1)}\right)\right| & \leq\left\|y^{(h)}\right\|_{N}\left\|\sum_{I_{n}}\left(x_{i}^{(n, j)}-x_{i}^{(n, 1)}\right) e^{i}\right\| \\
& <\tau \quad(n=1,2, \ldots ; j=2, \ldots, k+1 ; h=1, \ldots, k) .
\end{aligned}
$$

Next we show

$$
\rho\left(\sum_{J_{n}}\left(x_{i}^{(n, j)}-x_{i}^{(n, 1)}\right) e^{i}\right) \rightarrow 0 \quad(n \rightarrow \infty)
$$


By Lemma 2, for $i \in J_{n}$ and all $\left(x_{i}^{(n, j)}\right)_{j}$ have same sign; besides $\min _{j}\left|x_{i}^{(n, j)}\right| \leq \beta$, then

$$
M\left(\sum_{j} x_{i}^{(n, j)} /(k+1)\right) \leq(1-\delta) \sum_{j} M\left(x_{i}^{(n, j)}\right) /(k+1)
$$

Otherwise $\left(x_{i}^{(n, j)}\right)_{j}$ have different signs since $i \in J_{n}$. Say $x_{i}^{(n, 1)}, \ldots, x_{i}^{(n, p)}$ have same sign, $x_{i}^{(n, p+1)}, \ldots, x_{i}^{(n, k+1)}$ have same sign and $\sum_{j \leq p}\left|x_{i}^{(n, j)}\right| \geq \sum_{j>p}\left|x_{i}^{(n, j)}\right|$. Consider the $K+1$ numbers $x_{i}^{(n, 1)}, \ldots, x_{i}^{(n, p)}, 0, \ldots, 0$; obviously, they satisfy all the conditions in Lemma 2, for $b=M^{(-1)}(1), a=M^{-1}\left(\frac{1}{k+1}\right)$ and $m=K+1$. Therefore, there exists $\delta>0$ such that

$$
\begin{aligned}
M\left(\sum_{j \leq k+1} \frac{x_{i}^{(n, j)}}{k+1}\right) & <M\left(\sum_{j \leq p} \frac{x_{i}^{(n, j)}}{k+1}\right) \\
& \leq(1-\delta) \sum_{j \leq p} \frac{M\left(x_{i}^{(n, j)}\right)}{k+1} \\
& <(1-\delta) \sum_{j \leq k+1} \frac{M\left(x_{i}^{(n, j)}\right)}{k+1} .
\end{aligned}
$$

If (8) is not true, we can find a subsequence of $\{n\}$, again denoted by $\{n\}$, such that $\sum_{J_{n}} M\left(x_{i}^{(n, j)}-x_{i}^{(n, 1)}\right) \geq \sigma>0(n \geq 1)$. It follows from $M \in \delta_{2}$ that there exists a positive constant $c$ such that $\sum_{J_{n}} M\left(\frac{x_{i}^{(n, j)}-x_{i}^{(n, 1)}}{2}\right) \geq c \sigma \quad(n \geq 1)$. Therefore by (9)

$$
\begin{aligned}
0 & \leftarrow 1-\sum_{i \geq 1} M\left(\sum_{i \leq k+1} \frac{x_{i}^{(n, j)}}{k+1}\right) \\
& =\sum_{i \geq 1}\left\{\sum_{j \leq k+1}\left(M \frac{x_{i}^{(n, j)}}{k+1}\right)-M\left(\sum_{i \leq k+1} \frac{x_{i}^{(n, j)}}{k+1}\right)\right\} \\
& \geq \sum_{J_{n}}\left\{\sum_{j \leq k+1} M\left(x_{i}^{(n, j)}\right) /(k+1)-M\left(\sum_{i \leq k+1} x_{i}^{(n, j)} /(k+1)\right)\right\} \\
& >\delta \sum_{J_{n}}\left\{\sum_{j \leq k+1} M\left(x_{i}^{(n, j)}\right) /(k+1)\right\} \\
& \geq \delta \sum_{J_{n}} M\left(\frac{x_{i}^{(n, j)}-x_{i}^{(n, 1)}}{2}\right) /(k+1) \\
& \geq \frac{\delta c \sigma}{k+1} .
\end{aligned}
$$

This contradiction verifies (8). Hence from (3), for all $n$ large enough

$$
\left|\sum_{J_{n}} y_{i}^{(h)}\left(x_{i}^{(n, j)}-x_{i}^{(n, 1)}\right)\right| \leq\left\|y^{(h)}\right\|_{N}\left\|\sum_{J_{n}}\left(x_{i}^{(n, j)}-x_{i}^{(n, 1)}\right) e^{i}\right\|<\tau
$$

From (10), (7) and (5), it is easy to see that

$$
\Delta_{n}=\sup \left|\begin{array}{ccc}
\sum_{H_{n}} y_{i}^{(1)}\left(x_{i}^{(n, 2)}-x_{i}^{(n, 1)}\right) & \cdots & \sum_{H_{n}} y_{i}^{(1)}\left(x_{i}^{(n, k+1)}-x_{i}^{(n, 1)}\right) \\
\vdots & \vdots & \vdots \\
\sum_{H_{n}} y_{i}^{(k)}\left(x_{i}^{(n, 2)}-x_{i}^{(n, 1)}\right) & \cdots & \sum_{H_{n}} y_{i}^{(k)}\left(x_{i}^{(n, k+1)}-x_{i}^{(n, 1)}\right)
\end{array}\right|+O(\tau)
$$


Now, we show that $H_{n}$ contains no more than $K$ different numbers. In fact, otherwise, we may assume $1, \ldots, K+1 \in H_{n}, x_{1}^{(n, 2)}=\min _{j}\left|x_{1}^{(n, j)}\right|, x_{1}^{(n, 1)}=\max _{j}\left|x_{1}^{(n, j)}\right|$. From the definition of $H_{n}$, we have $x_{1}^{(n, 2)} \geq \beta$ and $x_{1}^{(n, 1)}-x_{1}^{(n, 2)} \geq \epsilon x_{1}^{(n, 1)}$, hence $x_{1}^{(n, 1)} \geq(1+\epsilon) \beta$. Combined with (4) we obtain a contradiction

$$
1=\rho\left(x^{(n, 1)}\right) \geq \sum_{j \leq k+1} M\left(x_{j}^{(n, 1)}\right)>M((1+\epsilon) \beta)+k M(\beta)>1 .
$$

We may assume, without loss of generality, $H_{n}=\{1, \ldots, k\}$ for all $n \geq 1$. (11) can be written as

$$
\Delta_{n}=\sup \left|\begin{array}{ccc}
\sum_{i \leq k} y_{i}^{(1)}\left(x_{i}^{(n, 2)}-x_{i}^{(n, 1)}\right) & \ldots & \sum_{i \leq k} y_{i}^{(1)}\left(x_{i}^{(n, k+1)}-x_{i}^{(n, 1)}\right) \\
\vdots & \vdots & \vdots \\
\sum_{i \leq k} y_{i}^{k}\left(x_{i}^{(n, 2)}-x_{i}^{(n, 1)}\right) & \ldots & \sum_{i \leq k} y_{i}^{k}\left(x_{i}^{(n, k+1)}-x_{i}^{(n, 1)}\right)
\end{array}\right|+O(\tau)
$$

Select a subsequence of $\left\{\Delta_{n}\right\}$, once more symbolized by $\left\{\Delta_{n}\right\}$, such that corresponding sequence $\left\{x_{i}^{(n, j)}\right\}_{n}$ converges, say the limit is $a_{i}^{j}(i=1,2, \ldots, k ; j=1, \ldots, k+1)$. Then from (12) it is easy to deduce

$$
\Delta_{n}=\sup \left|\begin{array}{ccc}
\sum_{i \leq k} y_{i}^{(1)}\left(a_{i}^{2}-a_{i}^{1}\right) & \ldots & \sum_{i \leq k} y_{i}^{(1)}\left(a_{i}^{k+1}-a_{i}^{1}\right) \\
\vdots & \vdots & \vdots \\
\sum_{i \leq k} y_{i}^{(k)}\left(a_{i}^{2}-a_{i}^{1}\right) & \ldots & \sum_{i \leq k} y_{i}^{(k)}\left(a_{i}^{k+1}-a_{i}^{1}\right)
\end{array}\right|+O(\tau)
$$

for $n$ large enough.

Notice that $\sum_{i \geq 1}\left\{\sum_{j \leq k+1} M\left(x_{i}^{(n, j)}\right) /(k+1)-M\left(\sum_{j \leq k+1} x_{i}^{(n, j)} /(k+1)\right)\right\} \rightarrow 0$, so $\sum_{j \leq k+1} M\left(x_{i}^{(n, j)}\right) /(k+1)-M\left(\sum_{j \leq k+1} x_{i}^{(n, j)} /(k+1)\right) \rightarrow 0$. Let $n \rightarrow \infty$; we have

$$
\sum_{j \leq k+1} M\left(a_{i}^{j}\right) /(k+1)=M\left(\sum_{j \leq k+1} a_{i}^{j} /(k+1)\right) \quad(i=1,2, \ldots, k)
$$

which shows $\left(a_{i}^{j}\right)_{j}$ are in the same interval on which $M(u)$ is linear, say

$$
M(u)=P_{i} u+Q_{i} ; \quad(i \leq k)
$$

From (6) and (8), when $n$ is large enough, we have $\sum_{I_{n} \cup J_{n}} M\left(x_{i}^{(n, j)}-x_{i}^{(n, 1)}\right)<(k+2) \epsilon(j \leq$ $k+1$ ) it follows from (2)

$$
\left|\sum_{I_{n} \cup J_{n}} M\left(x_{i}^{(n, j)}\right)-\sum_{I_{n} \cup J_{n}} M\left(x_{i}^{(n, 1)}\right)\right|<\tau \quad(j \leq k+1)
$$

Therefore, by $\rho\left(x^{(n, j)}\right)=1(j \leq k+1)$, we have $\left|\sum_{H_{n}} M\left(x_{i}^{(n, j)}\right)-\sum_{H_{n}} M\left(x_{i}^{(n, 1)}\right)\right|<\tau(j \leq$ $k+1)$. Let $n \rightarrow \infty$, we obtain $\left|\sum_{i \leq k} P_{i}\left(a_{i}^{j}-a_{i}^{1}\right)\right| \leq \tau$. Thus

$$
a_{i}^{j}-a_{1}^{1} \leq-\frac{1}{P_{1}} \sum_{i=2}^{k} P_{i}\left(a_{i}^{j}-a_{i}^{1}\right)+\frac{\tau}{P_{1}} \quad(j \leq k+1)
$$


Notice that $0<M^{\prime}(\beta) \leq M^{\prime}\left(a_{i}^{j}\right)=P_{i} \leq M^{\prime}\left(M^{-1}(1)\right)(i=1,2, \ldots, k)$, it is easy to deduce

$$
\Delta_{n}=\sup \left|\begin{array}{ccc}
\sum_{i=2}^{k}\left(y_{i}^{(1)}-y_{1}^{(1)} P i / P_{1}\right)\left(a_{i}^{2}-a_{i}^{1}\right) & \ldots & \sum_{i=2}^{k}\left(y_{i}^{(1)}-y_{1}^{(1)} P i / P_{1}\right)\left(a_{i}^{k+1}-a_{i}^{1}\right) \\
\vdots & \vdots & \vdots \\
\sum_{i=2}^{k}\left(y_{i}^{(k)}-y_{1}^{(k)} P i / P_{1}\right)\left(a_{i}^{2}-a_{i}^{1}\right) & \ldots & \sum_{i=2}^{k}\left(y_{i}^{(k)}-y_{1}^{(k)} P i / P_{1}\right)\left(a_{i}^{k+1}-a_{i}^{1}\right)
\end{array}\right|
$$

Finally, we expand the last determinant into $(K-1)^{k-1}$ many determinants of order $K$; each of them has the form

$$
\left|\begin{array}{ccc}
\left(y_{s_{1}}^{(1)}-y_{1}^{(1)} P_{s_{1}} / P_{1}\right)\left(a_{s_{1}}^{2}-a_{s_{1}}^{1}\right) & \ldots & \left(y_{s_{k}}^{(1)}-y_{1}^{(1)} P_{s_{k}} / P_{1}\right)\left(a_{s_{k}}^{k+1}-a_{s_{k}}^{1}\right) \\
\vdots & \vdots & \vdots \\
\left(y_{s_{1}}^{(k)}-y_{1}^{(k)} P_{s_{1}} / P_{1}\right)\left(a_{s_{1}}^{2}-a_{s_{1}}^{1}\right) & \ldots & \left(y_{s_{k}}^{(k)}-y_{1}^{(k)} P_{s_{k}} / P_{1}\right)\left(a_{s_{k}}^{k+1}-a_{s_{k}}^{1}\right)
\end{array}\right|
$$

Since each $s_{i}$ is between 2 and $K$, such determinant has at least two proportional columns, therefore it vanishes, i.e., (15) becomes $\Delta_{n}=O(\tau)$.

For any integer $K \geq 1$, we define $M(u)=u^{2}$ on $\left(-M^{-1}\left(\frac{1}{k+1}\right), M^{-1}\left(\frac{1}{k+1}\right)\right)$ and $M(u)=$ $2 M^{-1}(1 /(k+1))|u|-\left(M^{-1}(1 /(k+1))\right)^{2}$ otherwise; then it is easily verified by the theorem that $\ell_{M}$, generated by $M(u)$, is $k$ UR but not $(k-1)$ UR.

\section{REFERENCES}

1. F. Sullivan, A generalization of uniformly rotund Banach spaces, Canad. J. Math. 31(1979), 628-636.

2. A. Kaminska, On uniform convexity of Orlicz spaces, Indag. Math. A85(1982), 27-36.

3. W. Congxin, W. Tingfu, C. Shutao, W. Yuwen, Geometry of Orlicz spaces. Harbin, 1986.

4. J. Lindenstrauss, L. Tzafriri, Classical Banach spaces I. Springer-Verlag, 1977.

5. M. A. Krasnoselskii, Ya. B. Rutickii, Convex functions and orlicz spaces. Groningen, 1961.

\section{Department of Mathematics}

Harbin University of Science and Technology

Harbin, Heilongjiang

China

Harbin Teachers University

Harbin, Heilongjiang

China 